\title{
Maximum force levels in different positions of shoulder and elbow
}

\author{
Castro C. ${ }^{\mathrm{a}, *}$, De la Vega E. ${ }^{\mathrm{b}}$, Báez G. ${ }^{\mathrm{c}}$ and Carrasco F. ${ }^{\mathrm{d}}$. \\ ${ }^{a}$ Universidad Autónoma Indígena de México, Juárez 39, Mochicahui, Sinaloa, México. \\ bInstituto Tecnológico de Hermosillo, e delavega mx@yahoo.com, Sonora, México.

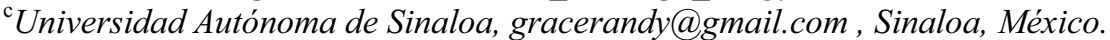 \\ ${ }^{\mathrm{d} I n s t i t u t o}$ Tecnológico de Hermosillo, fernandocarrasco2012@hotmail.com, Sonora, México.
}

\begin{abstract}
The number of occupational diseases in Mexico is alarming and clearly it is important to do studies with the purpose of improving the design of workstations. The objective of this research is to determine the maximum force levels in different positions of shoulder and elbow. An experiment was conducted with 16 subjects between 18 and 28 years old: 8 male and 8 female. We considered 16 different positions, working with the right and left arm to perform the tasks of pull and push. The tasks consisted of pushing or pulling a dynamometer for a period of 3 seconds as hard as possible. The results were presented in tables. The tables show the mean, standard deviation and range of force levels in different positions.
\end{abstract}

Keywords: Arms, Posture, Lateral, Frontal.

\section{Introduction}

Firms perform activities aimed at the prevention of occupational diseases; the results will be reflected in the production and productivity, as well as in better working environment and workers wellbeing. According to statistics from the Mexican Institute of Social Security (IMSS) during 2009 there were 4,101 new cases of occupational diseases in Mexico. No doubt this is alarming and clearly it is important to do studies with the purpose of improving the design of workstations.

The objective of this research is to determine the maximum force levels in different positions of shoulder and elbow.

\section{Materials and Method}

\subsection{Subject}

An experiment was conducted with 16 people between 18 and 28 years old: 8 male and 8 female. The participants were bachelor's degree students without manual material handling industrial abilities and they assure not to have any physical problem. Anthropometric measurements of the subjects were taken. The height was between $153.5 \mathrm{~cm}$ and $185.4 \mathrm{~cm}$ (mean $=166.6, \mathrm{SD}=9.2501 \mathrm{~cm})$ and the weight between $47.0 \mathrm{~kg}$ and $100.7 \mathrm{~kg}$ (mean $=66.55 \mathrm{~kg}$, SD. $=14.0639 \mathrm{~kg}$ ).

\subsection{Tasks}

The postures studied in the experiment were as follow: angles of $0^{\circ}, 20^{\circ}, 45^{\circ}$ y $90^{\circ}$ for lateral and frontal inclination. It considered 16 different positions, working with the right and left arm to perform the tasks of pull and push, resulting in participants doing 64 different tasks. The tasks consisted of pushing or pulling a dynamometer as hard as possible for a period of 3 seconds, the subjects would rest for 1 minute and then they would perform another pushing or pulling in a different position.

\footnotetext{
*Corresponding author. E-mail: klaudy 00@hotmail.com Phone: (52) 668 813-15-56. Fax: (52) 668 817-08-88.
} 1051-9815/12/\$27.50 C 2012 - IOS Press and the authors. All rights reserved 


\subsection{Procedure}

The participants received instructions about the tasks to be performed. They were instructed to work as hard as possible without straining themselves or becoming unusually tired, weakened, overheated or without of breath. Subjects performed the experiment in 4 sessions. The tasks per session were as follows:

- First session: $0^{0}$ angle of lateral inclination. 16 tasks (with $0^{\circ}, 20^{\circ}, 45^{\circ}$ and $90^{\circ}$ angle of frontal inclination; pushing and pulling; left and right arms), 3 replicates per task.

- Second session: $20^{\circ}$ angle of lateral inclination with 16 tasks.

- Third session: $45^{\circ}$ angle of lateral inclination with 16 tasks.

- Fourth session: $90^{\circ}$ angle of lateral inclination with 16 task.

The duration of each session was approximately 56 minutes. There was only one session per day in order to avoid fatigue in people.

\section{Results and discussions}

Tables 1 and 2 present the results of the experiment. For women the maximum values of force for the tasks of pulling and pushing were obtained at $45^{\circ}$ of lateral inclination. For men, the maximum values were presented at $45^{\circ}$ of lateral inclination for pulling and $0^{\circ}$ lateral inclination for pushing. $90^{\circ}$ of frontal inclination was the position associated with highest force for men and women in pushing and pulling. Men were stronger than women in the 64 tasks. Pulling required higher forces than pushing for men and women.

Table 1

Male maximum force

\begin{tabular}{|c|c|c|c|c|c|c|c|}
\hline Posture & $\begin{array}{l}\text { Arm } \\
\text { (Left, Right) }\end{array}$ & \multicolumn{3}{|c|}{ Pushing (Newton) } & \multicolumn{3}{|c|}{ Pulling (Newton) } \\
\hline Lateral $0^{\circ}$ - Frontal $0^{\circ}$ & $\mathrm{R}$ & 68.3557 & 24.5729 & $34.3210-100.6743$ & 76.8545 & 23.0019 & $36.2822-106.8854$ \\
\hline Lateral $0^{\circ}$ - Frontal $20^{\circ}$ & $\mathrm{R}$ & 62.1857 & 24.6062 & $16.6702-86.9459$ & 75.0561 & 26.1006 & $19.6120-98.7131$ \\
\hline \multirow[t]{2}{*}{ Lateral $0^{\circ}$ - Frontal $45^{\circ}$} & $\mathrm{L}$ & 66.2719 & 19.9935 & $29.4180-90.5417$ & 78.7745 & 23.5383 & $32.6863-102.6355$ \\
\hline & $\mathrm{R}$ & 65.6590 & 22.7803 & $25.8221-91.8489$ & 76.1593 & 22.9657 & $27.7833-95.7713$ \\
\hline Lateral $0^{\circ}$ - Frontal $90^{\circ}$ & $\mathrm{R}$ & 74.4844 & 30.3574 & $21.8997-120.9403$ & 93.3208 & 36.2077 & $23.8609-146.1094$ \\
\hline \multirow[t]{2}{*}{ Lateral $20^{\circ}$ - Frontal $0^{\circ}$} & $\mathrm{L}$ & 64.8824 & 21.8742 & $29.7445-87.5999$ & 80.4494 & 26.2320 & $33.3404-105.5773$ \\
\hline & $\mathrm{R}$ & 60.5109 & 23.3402 & $19.9385-86.2928$ & 78.3254 & 24.9043 & $26.4762-99.3671$ \\
\hline \multirow[t]{2}{*}{ Lateral $20^{\circ}$ - Frontal $20^{\circ}$} & $\mathrm{L}$ & 65.9042 & 25.6760 & $20.5926-96.0988$ & 78.0803 & 26.6017 & $26.4762-105.9048$ \\
\hline & $\mathrm{R}$ & 67.4163 & 22.8293 & $26.1487-94.7907$ & 76.8947 & 23.0117 & $26.8027-95.7713$ \\
\hline Lateral $20^{\circ}$ - Frontal $45^{\circ}$ & $\mathrm{L}$ & 70.1943 & 28.0334 & $23.2069-104.5967$ & 76.6094 & 22.6538 & $33.3404-98.7131$ \\
\hline \multirow[t]{2}{*}{ Lateral $20^{\circ}$ - Frontal $90^{\circ}$} & $\mathrm{L}$ & 66.8848 & 24.0933 & $32.6863-99.3671$ & 85.1073 & 21.9478 & $44.4535-112.1149$ \\
\hline & $\mathrm{R}$ & 70.1129 & 22.6597 & $36.9353-104.9242$ & 85.1073 & 19.6287 & $48.0494-109.1731$ \\
\hline \multirow[t]{2}{*}{ Lateral $45^{\circ}$ - Frontal $0^{\circ}$} & $\mathrm{L}$ & 64.5970 & 23.8266 & $25.4956-92.8295$ & 82.3704 & 27.4539 & $33.3404-118.6526$ \\
\hline & $\mathrm{R}$ & 62.3907 & 22.9931 & $25.8221-86.2928$ & 81.8389 & 26.9538 & $28.4374-107.5385$ \\
\hline \multirow[t]{2}{*}{ Lateral $45^{\circ}$ - Frontal $20^{\circ}$} & $\mathrm{L}$ & 62.0632 & 21.3673 & $25.1681-89.8877$ & 79.4286 & 27.1783 & $24.8415-112.1149$ \\
\hline & $\mathrm{R}$ & 60.6746 & 21.6615 & $25.1681-96.0988$ & 79.5914 & 22.2253 & $29.4180-98.7131$ \\
\hline \multirow[t]{2}{*}{ Lateral $45^{\circ}$ - Frontal $45^{\circ}$} & $\mathrm{L}$ & 59.1214 & 19.1766 & $22.8803-86.6193$ & 80.8583 & 24.7651 & $31.0517-117.3445$ \\
\hline & $\mathrm{R}$ & 65.4551 & 24.9033 & $25.1681-107.2119$ & 81.3898 & 23.4216 & $32.6863-111.7884$ \\
\hline \multirow[t]{2}{*}{ Lateral $45^{\circ}$ - Frontal $90^{\circ}$} & $\mathrm{L}$ & 61.5327 & 24.2826 & $24.1875-104.5967$ & 82.1253 & 26.5311 & $30.3986-118.6526$ \\
\hline & $\mathrm{R}$ & 70.4394 & 29.5543 & $24.5150-126.1699$ & 83.6775 & 25.2289 & $33.9935-112.4415$ \\
\hline \multirow[t]{2}{*}{ Lateral $90^{\circ}$ - Frontal $0^{\circ}$} & $\mathrm{L}$ & 64.1067 & 27.7951 & $20.9191-105.9048$ & 74.8521 & 33.1207 & $22.8803-127.4780$ \\
\hline & $\mathrm{R}$ & 62.3093 & 26.2124 & $21.8997-102.9630$ & 73.7490 & 31.3262 & $25.4956-120.9403$ \\
\hline \multirow[t]{2}{*}{ Lateral $90^{\circ}$ - Frontal $20^{\circ}$} & $\mathrm{L}$ & 59.5715 & 25.4270 & $18.3039-95.1182$ & 77.6714 & 24.8886 & $36.2822-116.6914$ \\
\hline & $\mathrm{R}$ & 61.6552 & 24.4905 & $19.9385-97.4059$ & 74.8933 & 25.9682 & $32.0323-109.8272$ \\
\hline \multirow[t]{2}{*}{ Lateral $90^{\circ}$ - Frontal $45^{\circ}$} & $\mathrm{L}$ & 63.3713 & 25.4377 & $20.9191-98.0600$ & 81.7575 & 31.7293 & $26.1487-118.6526$ \\
\hline & $\mathrm{R}$ & 67.0485 & 26.2958 & $26.4762-100.3477$ & 79.1835 & 28.7737 & $26.4762-110.8078$ \\
\hline \multirow[t]{2}{*}{ Lateral $90^{\circ}$ - Frontal $90^{\circ}$} & $\mathrm{L}$ & 77.0585 & 35.2859 & $22.5538-128.1311$ & 89.0708 & 27.7490 & $46.7413-124.2087$ \\
\hline & $\mathrm{R}$ & 77.5900 & 33.8278 & $26.8027-115.7108$ & 85.1484 & 28.7924 & $41.8383-130.4198$ \\
\hline
\end{tabular}


Table 2

Female maximum force

\begin{tabular}{|c|c|c|c|c|c|c|c|}
\hline \multirow{2}{*}{ Posture } & \multirow{2}{*}{ Arm } & \multicolumn{3}{|c|}{ Pushing (Newton) } & \multicolumn{3}{|c|}{ Pulling (Newton) } \\
\hline & & Mean & SD & Range & Media & SD & Range \\
\hline \multirow[t]{2}{*}{ Lateral $0^{\circ}$ - Frontal $0^{\circ}$} & $\mathrm{L}$ & 37.2628 & 16.6133 & $23.5344-70.6032$ & 42.8199 & 7.4045 & $30.3986-50.3371$ \\
\hline & $\mathrm{R}$ & 35.8321 & 14.1785 & $24.1875-64.7196$ & 43.3504 & 9.7746 & $31.3792-55.2401$ \\
\hline \multirow[t]{2}{*}{ Lateral $0^{\circ}$ - Frontal $20^{\circ}$} & $\mathrm{L}$ & 32.7677 & 12.2163 & $19.2845-51.6443$ & 36.8951 & 10.3179 & $18.6314-53.2789$ \\
\hline & $\mathrm{R}$ & 32.2372 & 11.3769 & $18.6314-53.2789$ & 39.2642 & 10.1129 & $27.1293-52.6249$ \\
\hline \multirow[t]{2}{*}{ Lateral $0^{\circ}$ - Frontal $45^{\circ}$} & $\mathrm{L}$ & 37.9159 & 15.5278 & $24.1875-69.6226$ & 41.3892 & 11.9751 & $22.8803-56.5473$ \\
\hline & $\mathrm{R}$ & 36.4862 & 12.6282 & $25.8221-57.8554$ & 43.3504 & 12.8978 & $27.4568-62.1043$ \\
\hline \multirow[t]{2}{*}{ Lateral $0^{\circ}$ - Frontal $90^{\circ}$} & $\mathrm{L}$ & 45.8019 & 12.3683 & $32.0323-67.9879$ & 55.1588 & 11.9104 & $41.8383-80.7357$ \\
\hline & $\mathrm{R}$ & 47.7219 & 12.5576 & $30.7251-70.2757$ & 57.8142 & 15.8945 & $36.6087-92.8295$ \\
\hline \multirow[t]{2}{*}{ Lateral $20^{\circ}$ - Frontal $0^{\circ}$} & $\mathrm{L}$ & 36.7313 & 13.6823 & $15.0355-55.2401$ & 47.8445 & 18.9030 & $15.3621-76.8133$ \\
\hline & $\mathrm{R}$ & 37.3854 & 13.4430 & $16.3427-55.5667$ & 51.0726 & 22.6313 & $16.6702-86.6193$ \\
\hline \multirow[t]{2}{*}{ Lateral $20^{\circ}$ - Frontal $20^{\circ}$} & $\mathrm{L}$ & 39.1014 & 14.8031 & $15.0355-59.4891$ & 46.8237 & 17.1105 & $13.4009-70.6032$ \\
\hline & $\mathrm{R}$ & 39.2240 & 13.5450 & $20.5926-53.9330$ & 49.0702 & 20.6456 & $15.0355-80.0817$ \\
\hline \multirow[t]{2}{*}{ Lateral $20^{\circ}$ - Frontal $45^{\circ}$} & $\mathrm{L}$ & 40.0408 & 16.4780 & $16.3427-62.1043$ & 50.3371 & 17.4782 & $18.6314-72.2369$ \\
\hline & $\mathrm{R}$ & 39.5505 & 16.4780 & $17.6508-58.5085$ & 48.2534 & 20.2445 & $16.6702-75.8327$ \\
\hline \multirow[t]{2}{*}{ Lateral $20^{\circ}$ - Frontal $90^{\circ}$} & $\mathrm{L}$ & 41.3892 & 16.6163 & $20.2651-63.0849$ & 51.8080 & 17.2439 & $26.1487-80.4092$ \\
\hline & $\mathrm{R}$ & 41.3490 & 17.7930 & $24.5150-64.7196$ & 51.1138 & 17.2556 & $28.7639-83.3510$ \\
\hline \multirow[t]{2}{*}{ Lateral $45^{\circ}$ - Frontal $0^{\circ}$} & $\mathrm{L}$ & 39.7143 & 10.4787 & $27.7833-58.8360$ & 55.2813 & 20.6573 & $37.2628-90.2152$ \\
\hline & $\mathrm{R}$ & 42.4923 & 11.3230 & $30.3986-60.4697$ & 55.9344 & 22.2106 & $38.8965-95.1182$ \\
\hline \multirow[t]{2}{*}{ Lateral $45^{\circ}$ - Frontal $20^{\circ}$} & $\mathrm{L}$ & 41.5931 & 15.2915 & $21.8997-62.7584$ & 55.9756 & 22.3714 & $28.4374-91.8489$ \\
\hline & $\mathrm{R}$ & 40.8577 & 14.6364 & $19.2845-59.1625$ & 56.8336 & 22.0253 & $32.0323-91.5223$ \\
\hline \multirow[t]{2}{*}{ Lateral $45^{\circ}$ - Frontal $45^{\circ}$} & $\mathrm{L}$ & 40.6949 & 15.0453 & $24.1875-68.6420$ & 54.2184 & 21.7036 & $25.8221-87.2734$ \\
\hline & $\mathrm{R}$ & 41.8795 & 15.4003 & $25.4956-63.7390$ & 55.9354 & 20.5808 & $32.6863-87.2734$ \\
\hline \multirow[t]{2}{*}{ Lateral $45^{\circ}$ - Frontal $90^{\circ}$} & $\mathrm{L}$ & 43.3504 & 13.0371 & $28.1099-65.0461$ & 56.1796 & 15.8524 & $36.6087-82.0429$ \\
\hline & $\mathrm{R}$ & 44.3310 & 16.5045 & $27.1293-73.5450$ & 57.0788 & 18.9128 & $39.8771-95.4447$ \\
\hline \multirow[t]{2}{*}{ Lateral $90^{\circ}$ - Frontal $0^{\circ}$} & $\mathrm{L}$ & 37.7933 & 9.9698 & $21.8997-50.9912$ & 49.8880 & 18.4902 & $21.2457-84.0041$ \\
\hline & $\mathrm{R}$ & 37.0177 & 10.7856 & $21.8997-50.6637$ & 45.1478 & 16.8487 & $20.9191-73.8715$ \\
\hline \multirow[t]{2}{*}{ Lateral $90^{\circ}$ - Frontal $20^{\circ}$} & $\mathrm{L}$ & 35.9145 & 11.2779 & $21.8997-58.5085$ & 45.6793 & 15.3444 & $23.2069-78.1205$ \\
\hline & $\mathrm{R}$ & 35.6281 & 11.7309 & $22.5538-53.9330$ & 45.8431 & 16.5859 & $29.7445-78.1205$ \\
\hline \multirow[t]{2}{*}{ Lateral $90^{\circ}$ - Frontal $45^{\circ}$} & $\mathrm{L}$ & 39.5094 & 9.4255 & $26.1487-52.9524$ & 48.1720 & 15.0189 & $24.1875-74.1981$ \\
\hline & $\mathrm{R}$ & 37.6707 & 11.2847 & $25.1681-53.6055$ & 48.0896 & 16.3731 & $26.1487-76.1593$ \\
\hline \multirow[t]{2}{*}{ Lateral $90^{\circ}$ - Frontal $90^{\circ}$} & $L$ & 40.4086 & 13.7206 & $24.1875-61.1237$ & 60.2245 & 17.7940 & $30.3986-90.5417$ \\
\hline & $R$ & 42.7787 & 12.2232 & $30.3986-62.1043$ & 64.0244 & 19.9689 & $36.6087-96.4253$ \\
\hline
\end{tabular}

\section{References}

[1] B. Le Veau, Biomecánica del movimiento humano, Trillas ed., México, 2008, pp. 208-215.

[2] D.B. Chaffin; G.B. Anderson and B.J. Martin, Occupational Biomechanics, Wiley Interscience ed., Massachusetts, 1999, pp. 315-349.

[3] F.J. Llaneza, Ergonomía y psicosociología aplicada: manual para la formación del especialista, Lex Nova ed, España, 2009, pp.157-189.

[4] J.A. Cruz and G.A. Garnica, Principios de ergonomía, ECOE ed., Colombia, 2006, pp. 38-48.

[5] M. Rodríguez, Ergonomía básica aplicada a la medicina del trabajo, Díaz de Santos ed., España, 1994, pp. 19-35.

[6] P.R. Mondelo, E. Gregori, J. Blasco and P. Barrau, Ergonomía 3: Diseño de puestos de trabajo, Universidad Politécnica de Cataluña ed., España, 1998, pp. 105-125.

[7] S. Konz, Diseño de sistemas de trabajo, Limusa ed., México, 2005, pp. 305-306. 